\title{
Das Germanistik- bzw. DaF-Studium in der Türkei
}

\author{
Probleme und Lösungsvorschläge
}

\section{Tahir Balcı}

Seit Jahren befindet sich das Germanistik- bzw. das DaF-Studium in einer Krise, die die Motivation von Lehrenden und Lernenden zum Schwinden bringt. Um das Interesse am Deutschstudium erneut zu wecken, werden seit einigen Jahren einzelne Versuche gestartet (Kula 1991; Polat 1996). Da diese jedoch über punktuelle Änderungen im Curriculum nicht hinausgehen oder nicht praktiziert werden, steht keine Besserung in Aussicht.

Das türkische Erziehungsministerium stellt etwa seit 1985 keine Deutschlehrer mehr an. Die vor diesem Zeitpunkt angestellten Deutschlehrer werden als Springer eingesetzt und müssen fachfremde Themen unterrichten.

Um Abhilfe zu schaffen, sehen wir uns gezwungen, das Germanistikstudium entsprechend den Anforderungen des Arbeitsmarktes von Grund auf umzustrukturieren.

Um die Anforderungen des Arbeitsmarktes festzustellen, haben wir eine schriftliche Umfrage durchgeführt. An 35 private und öffentliche Institutionen (Banken, Ministerien, Holdings) wurde ein Umfragebogen geschickt, durch den festgestellt werden sollte, was für Personal sie be- schäftigen, welche Sach- und Fachkenntnisse also jemand erworben haben muß, um Aussicht auf eine Arbeit zu haben. Außerdem haben wir zwei Monate lang die Zeitungsanzeigen verschiedener Institutionen ausgeschnitten, aus denen die Entwicklungstendenzen des Arbeitsmarktes hervorgehen. Folgendes hat sich ergeben:

a) Unabhängig von Fachkenntnissen sind sehr gute Fremdsprachenkenntnisse eine Voraussetzung für eine Anstellung. Die Chancen vergrößern sich, wenn der Bewerber über Kenntnisse in einer zweiten Fremdsprache verfügt.

b) Neben einer sehr guten Fach- und Fremdsprachenkompetenz ist fachsprachliches Wissen auf den Gebieten Jura, Wirtschaft, Kunst und Kultur eine weitere Bedingung.

c) Es besteht ein großer Bedarf an simultanen Übersetzern.

d) Es soll auch die Fähigkeit erworben werden, ausländischen Beschäftigten Türkischunterricht zu erteilen.

e) Für den Tourismus-Sektor braucht man dringend Personal, das über sehr gute Fach- und Fremdsprachenkenntnisse verfügt. 
Diese Ergebnisse können wir in Punkten zusammenfassen:

1) Weil sich die Türkei der europäischen Zollunion angeschlossen hat und auch die Mitgliedschaft in der EU bezweckt, haben die Beziehungen zu den europäischen Ländern zugenommen. Dementsprechend braucht man (vor allem simultane) Übersetzer, die die Verständigung zwischen den Partnern ermöglichen und die juristischen bzw. Handelsbestimmungen ins Türkische übertragen können. Um die Nachfrage nach qualifizierten Übersetzern zu decken, wurden schon an der Bogaziçi-, Hacettepe-, Istanbul- und Mersin-Universität Abteilungen für Übersetzen-Dolmetschen eingerichtet.

2) Obwohl die Türkei aufgrund ihrer historischen Monumente bzw. ihrer Naturschönheiten und auch des geringen Preisniveaus touristischer Dienstleistungen das bevorzugte Reiseland vieler Europäer, aber besonders der Deutschen ist, mangelt es ihr an qualifizierten Arbeitskräften im Tourismus-Bereich.

3) Wenn man den türkischen Gegebenheiten Rechnung tragen will, scheint es ein pragmatischer Ausweg zu sein, die Ausbildung des Tourismus-Personals germanistischen Abteilungen zu übertragen. Die Kombination von DaF-Studium und Tourismus ist in zweierlei Hinsicht notwendig: Auf diese Weise kann nämlich nicht nur die Krise, in der sich DaF befindet, überwunden werden, sondern partiell auch diejenige des türkischen Tourismus. Ammon (1991) bezeichnet ebenfalls diese Verbindung als einen denkbaren Ausweg aus der gegenwärtigen Krise.

Auf dem Tourismus-Markt kommt der deutschen Sprache besondere Bedeutung $\mathrm{zu}$.
1) Durch die starke Zunahme der Freizeit ändert sich auch das Verhalten der Menschen. Die durchschnittliche Jahresarbeitszeit betrug 19602100 Stunden; vermutlich werden es im Jahr 2000 um die 1500 Stunden sein. Man erwartet also ein Wachstum des Freizeit- und Urlaubsmarktes (Studiengang München 1994: 4).

2) Deutsche Staatsbürger haben eine unübertreffliche Reiselust. Daß mehr als $65 \%$ der Bundesbürger über 14 Jahre mindestens eine Urlaubsreise im Jahr unternehmen und mehr als 40 Milliarden DM jährlich für Reisen ausgegeben werden bzw. dies etwa einem Fünftel der weltweiten Ausgaben für Tourismus entspricht, ist ein triftiger Grund dafür (Opaschowski 1989: 29).

3) Binnen der ca. 30-jährigen Geschichte der drei Generationen türkischer Staatsbürger in Deutschland, deren Anzahl zwei Millionen übersteigt, sind zwischen Deutschen und Türken zahlreiche soziokulturelle Beziehungen entstanden, die die Deutschen dazu motivieren, Leben und Kultur ihrer Mitmenschen vor Ort kennenzulernen.

4) Nach den Statistiken des türkischen Ministeriums für Tourismus kommt die überwältigende Mehrheit der Touristen, die die Türkei als Reiseland wählen, aus deutschsprachigen Ländern, hauptsächlich aus Deutschland. Auch die Anfang 1996 von N. Yavus vorgelegte Magisterarbeit, die die Möglichkeiten des Beitrags des Germanistikstudiums in der Türkei zum Tourismus erforschte, hat ergeben, daß über $80 \%$ der in die Türkei Reisenden Muttersprachler des Deutschen sind.

5) Rückkehrerkinder bilden das studentische Potential für DaF. Einerseits wollen sie ihre noch vorhande- 
nen guten Deutsch(land)kenntnisse bewahren und lebendig erhalten, andererseits möchten sie ihr Wissen um Kenntnisse ergänzen, die auf dem Arbeitsmarkt gefragt sind. Gerade der Tourismus ist der andere Kenntnisbereich. Denn er setzt die Erfüllung zweier Bedingungen voraus: sehr gute Deutschkenntnisse und Qualifikation auf dem TourismusSektor. Bei unseren Studenten ist die erste Bedingung meistens weitgehend erfüllt. Was ihnen fehlt, sind touristische Fachkenntnisse.

6) Untersuchungen ergeben (Agaoḡlu 1993: 310; Bir 1993: 273), daß die im Tourismus Beschäftigten zu 90 \% keine Fachkenntnisse besitzen. Sie sind entweder in den Fachhochschulen für Tourismus und Hoteladministration gewissermaßen als Buchhalter und Finanzfachleute ausgebildet und verfügen kaum über theoretische und praktische Tourismus- und Fremdsprachenkenntnisse oder sie haben trotz sehr guter Sprachbeherrschung keine Ausbildung in Touristik. Das ist der Fall bei türkischen DaF-Absolventen, die den Bedarf an Personal für den Tourismus-Bereich de facto decken.

7) Da die vorhandenen TourismusFachhochschulen die nachgefragten Qualifikationen nicht vermitteln, bieten verschiedene Vereine $\mathrm{zu}$ diesem Zweck Kurse an. Reiseleiter bzw. Reisekaufleute werden in dreibis sechsmonatigen Kursen mit 3-4 Wochenstunden ausgebildet. Aber die Probleme sind $\mathrm{zu}$ vielfältig, als daß man sie durch Kurse in den Griff bekommen könnte. Sie müssen vielmehr durch eine umfassende universitäre Ausbildung gelöst werden, die Sprach- und Fachausbildung professionell miteinander verbindet.
8) Daß die oben genannten Fachhochschulen den Bedarf an TourismusPersonal nicht decken können, liegt am Inhalt ihrer Lehrveranstaltungen, die den tatsächlichen Anforderungen des Fremdenverkehrs-Sektors nicht angemessen sind. Wie wäre es sonst zu erklären, daß von den derzeit über zehntausend Absolventen derartiger Fachhochschulen nur $20 \%$ im Tourismus eine Anstellung finden, meist aber als freiberufliche Buchhalter arbeiten (Hac loglu 1993: 91)? Hier zum Beispiel der Lehrplan des 1. Semesters der Fachhochschule der Mersin-Universität: Einführung in die Wirtschaft, Einführung in die Mathematik, Einführung in die Betriebslehre, Sozialpsychologie, Englisch, Hoteladministration, Atatürkische Reformen, Musik und Gymnastik.

9) Der Bedarf des touristischen Arbeitsmarktes wird daher fast nur mit ausgebildeten Germanisten (bzw. DaFAbsolventen) gedeckt, die die freien Stellen einnehmen. Wenn man also DaF mit Touristik kombiniert, werden die Studenten nicht mehr nur studieren, um später irgendein Diplom in der Tasche zu haben, sondern wegen der sich abzeichnenden Berufschancen. Die intendierte Kombination würde $\mathrm{m}$. E. zu einem bevorzugten Studiengang in der Türkei. Die Motivation zum Deutschlernen würde automatisch enorm steigen.

10) Die wichtigste Voraussetzung für eine Tätigkeit im Tourismusbereich ist die sehr gute Beherrschung der deutschen Sprache. Im Widerspruch dazu ergaben diesbezügliche Untersuchungen, daß die Absolventen der Tourismus-Fachhochschulen nicht in der Lage sind, in einer Fremdsprache angemessen mündlich oder schrift- 
lich zu kommunizieren. Unterrichtssprache ist dort entweder Türkisch (mit Englisch als 1. Fremdsprache) oder Englisch mit Deutsch oder Französisch als Wahlfach. Wie schon erwähnt, hat diese Ausbildungsvariante bisher keinen Erfolg gezeigt. Sie ist auch in Zukunft zum Scheitern verurteilt.

Die türkischen Gegebenheiten fordern die Kombination von DaF und Touristik. Französisch und Englisch sollen als Wahlfächer fungieren.

11) Nach neuesten Forschungen wird man in der Türkei in den nächsten fünf Jahren 80.000 Tourismus-Fachleute brauchen (vgl. Dall 1993: 321). Für Absolventen des geplanten Studienganges ist also ein großer Arbeitsmarkt vorhanden.

Fazit: Die Einrichtung einer Unterabteilung durch das Germanistische Seminar der Pädagogischen Fakultät der Çukurova-Universität, der bei einem persönlichen Gespräch auch der Rektor zugestimmt hat, ist ein Desiderat. Die vorgesehene Unterabteilung wird ausschließlich Studenten aufnehmen, die ihre Deutschkenntnisse bei der sogenannten interuniversitären Aufnahmeprüfung nachgewiesen haben. Das erste Jahr wird als Vorbereitungsklasse fundierte Deutschkenntnisse vermitteln und auf das eigentliche Studium vorbereiten. Das Curriculum der weiteren acht Semester muß noch in Zusammenarbeit mit Fachleuten aus dem Tourismus-Bereich erarbeitet werden.

\section{Literatur}

Agaoḡlu, K.: »Turizm Egitiminin Batı Avrupa Olkeleri ile Karşilaşturmali Konumu ve Türkiye Uygulamasında Yaşanılan Tikanıklkklar«. In: Turizm Egitimi; Turizm Bak. 1993, 291-314.

Ammon, U.: Die internationale Stellung der deutschen Sprache. Berlin: de Gruyter, 1991.

Bir, A. u. a.: »Turizm Egitiminde Uzaktan Egitim Teknolojisi Uygulaması ve Degerlendirmesi«. In: Turizm Egitimi; Turizm Bak. 1993, 273-289.

Boyacı, C.: »Üniversite Düzeyinde Turizm Egitimi Veren Yükseköğretim Kurumlarında Ögretim Elemanı Yetiștirme Sorunları ve Çözüm Önerileri«. In: Turizm Egitimi; Turizm Bak. 1993, 121-123.

Dallı, Ö.: »Turizm Egitiminde TUGEV' in Yeri«. In: Turizm Egitimi; Turizm Bak. 1993, 321-324.

Hacıoglu, N.: »Yüksekögrretimde Mesleki Turizm Egitimi Geliștirme Perspektifleri«. In: Turizm Egitimi; Turizm Bak. 1993, 91-100.

Kula, O.B.: »Hochschul- und fachdidaktische Überlegungen zur Analyse und Reform der Germanistik und Deutschlehrerausbildung in der Türkei«. In: Ankaraner Beiträge zur Germanistik. Ankara/ Istanbul 1991, 13-23.

Opaschowski, H. W.: Tourismusforschung. Opladen: Leske + Budrich, 1989 .

Polat, T.: »Der interkulturelle Ansatz und einige Überlegungen zum Deutschunterricht in der Türkei«. In: Almanca Dil Dergisí. AÖD Yayını. 1996, 125-133.

Studiengang Tourismus. Fachhochschule München, 1994.

Turizm Eḡitimi. Turizm Bak. Yay. Ankara 1993.

Turizm istatistikleri 1992. DIE Matbaasi. Ankara 1994. 